\title{
Comparación de la abundancia, estructura de tallas y fecundidad de Voluta musica (Caenogastropoda: Volutidae) en tres sitios de la costa norte de la Península de Araya, Venezuela
}

\author{
Ana Carolina Peralta ${ }^{1}$, Patricia Miloslavich ${ }^{1} \&$ Gregorio Bigatti $^{2}$ \\ 1. Universidad Simón Bolívar, Departamento de Estudios Ambientales, Centro de Biodiversidad Marina, Laboratorio de \\ Biología Marina, Apdo. 89000 Caracas 1086-A Venezuela, Telef.: 0058-212-9063052; \\ aperalta@usb.ve,pmilos@usb.ve \\ 2. Centro Nacional Patagónico, Pto. Madryn, Argentina 0054-2965-451024 int. 273; gbigatti@ cenpat.edu.ar
}

\author{
Recibido 20-VII-2011. Corregido 13-XII-2011. Aceptado 20-XII-2011.
}

\begin{abstract}
Abundance, size structure and fecundity of Voluta musica (Caenogastropoda: Volutidae) in three sites of the north coast of Araya Peninsula, Venezuela. Considering the intensive artisanal fishing activity and the consequent carrion discard found at Isla Caribe, in relation to other two sites with no intensive artisanal fishing activity, we expect different effects on some features of $V$. musica life history (larger egg capsules, larger organisms, higher abundance of adult organisms). In this paper we compare some population parameters of Voluta musica at three localities in the north coast of the Araya Peninsula in Venezuela under different fishing exploitation regimes. The samples were taken monthly during 2008 and 2009 at Isla Caribe, Isla Lobos and Bajo Cuspe. At each site, samples were taken within three areas of $40 \mathrm{~m}^{2}$. The abundance of $V$. musica ranged between $5 \mathrm{ind} / 120 \mathrm{~m}^{2}$ to $30 \mathrm{ind} / 120 \mathrm{~m}^{2}$ with significant differences between sites $(\mathrm{F}=7.77 ; \mathrm{p}<0,01)$. Organisms from Isla Caribe were larger in size $(\mathrm{p}=0,045)$, than those in the other two sites. There is a significant differences in the number of egg capsules between sites and between months, and there is clear evidence that Isla Caribe has the largest abundance of egg capsules $(\mathrm{p}<0,01)$ suggesting that the extra feeding source (carrion) found at Isla Caribe could have a positive effect on the reproductive potential of the V. musica population at this site. Rev. Biol. Trop. 60 (Suppl. 1): 165-172. Epub 2012 March 01.
\end{abstract}

Key word: Volutidae, artisanal fisheries, egg capsules, fecundity, South Caribbean.

Voluta musica (Linné, 1758) es un gasterópodo marino endémico del Caribe sur que se encuentra en el Libro Rojo de la Fauna Venezolana en la categoría "Insuficientemente conocido" (Rodríguez \& Rojas-Suárez 2008). Es una especie gonocórica, de desarrollo directo, que adhiere sus ovicápsulas al substrato, usualmente en la parte interna de conchas vacías de bivalvos (e.g., Anadara sp., Atrina sp., Pinna sp.). Las cápsulas son hemi-esféricas y miden aproximadamente $18 \mathrm{~mm}$ de diámetro basal. Hay de 3 a 5 embriones dentro de cada cápsula, los cuales se alimentan del fluido intracapsular (Gibson-Smith 1973, Penchaszadeh \& Miloslavich 2001).
Isla Caribe, Isla Lobos y Bajo Cuspe son tres sitios ubicados al noreste de la Península de Araya donde se hallan poblaciones de V. musi-

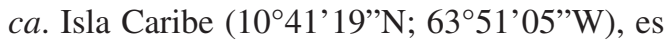
un pequeño islote donde existe un asentamiento permanente de pescadores artesanales con un tráfico marino relativamente alto llevado a cabo por seis botes de $6 \mathrm{~m}$ de eslora cada uno, adicionalmente es una zona de paso de otras embarcaciones pesqueras y turísticas. Los mismos diariamente realizan faenas de pesca, descartando especies no comerciales de peces, bivalvos y cangrejos en zonas someras de la playa. Cabe destacar que los botes utilizados están pintados con pinturas antiincrustantes, 
que sumado a los desechos de los motores (hidrocarburos) son un factor de contaminación que afecta a los caracoles produciendo imposex en hembras de $V$. musica (A. C. Peralta, en

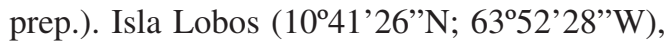
es otro pequeño islote localizado a $2 \mathrm{~km}$ del primero. El ecosistema marino es semejante al de Isla Caribe, pero el tráfico marino es de bajo a nulo ya que al sitio llega un bote de $6 \mathrm{~m}$ de eslora por semana y actualmente no hay asentamientos, botes permanentes ni descartes diarios de pesca, donde A. C. Peralta (en prep.) encontraron una baja incidencia de imposex de $4 \%$. Bajo Cuspe $\left(10^{\circ} 43^{\prime} 53^{\prime \prime} \mathrm{N}\right.$; 63⒌ $\left.51^{\prime} 10^{\prime \prime} \mathrm{W}\right)$, es un sitio que se encuentra a $3 \mathrm{~km}$ de Isla Caribe e Isla Lobos y no presenta botes permanentes ni descartes diarios de la pesca artesanal y en el cual no se han registrado casos de imposex (A.C. Peralta, en prep.) (Observ. pers.).

En Isla Caribe, la presencia de la pesca artesanal con frecuencia diaria genera una fuente alóctona de alimento por descarte pesquero, el cual es aprovechado por las especies carroñeras (gasterópodos, crustáceos, peces, etc.). V. musica tiene tanto hábitos carroñeros como depredatorios (von Cosel 1976), bajo este escenario se pretende estudiar el efecto que tendría una fuente adicional de alimento sobre algunos rasgos de la historia de vida en las poblaciones de esta especie. Esta mayor disposición de alimento podría estar relacionada con una abundancia mayor, hembras de tallas mas grandes y un mayor numero de cápsulas por hembra en relación a Isla Lobos y Bajo del Cuspe donde no existe tal fuente de alimento y en donde $V$. musica se alimenta principalmente por depredación, con un mayor costo energético (A.C. Peralta, obs. pers.).

El objetivo del presente trabajo es conocer la abundancia, estructura de talla y fecundidad de $V$. musica en tres sitios de la costa norte de la Península de Araya en las que existen actividades de pesca diferenciales.

\section{MATERIALES Y MÉTODOS}

Se realizaron muestreos mensuales entre marzo de 2008 y diciembre de 2009 en Isla
Caribe, Isla Lobos y Bajo Cuspe. En cada sitio se delimitaron tres transectas de $20 \mathrm{~m} \times 2 \mathrm{~m}$ $\left(40 \mathrm{~m}^{2}\right)$ paralelas a la línea de costa sobre la zona habitada por $V$. musica, a una profundidad de 1,5 m para Isla Caribe e Isla Lobos y a nueve metros en Bajo Cuspe. Se contaron todas las ovicápsulas y los individuos encontrados a lo largo de cada transecta y se determinó la talla y el sexo de cada uno. Sabiendo que las hembras comienzan a madurar a $10 \mathrm{~s} 5 \mathrm{~mm}$ de longitud de la concha (A.C. Peralta, en prep) se registró la proporción de hembras mayores a $60 \mathrm{~mm}$ en cada sitio y en cada mes, con el fin de realizar una estimación indirecta de la fecundidad como una relación entre el número de ovicápsulas encontradas/hembra en cada mes. Se evaluaron las diferencias en la abundancia de hembras maduras a lo largo de los meses del año a través de un análisis de la varianza.

Se registró mensualmente la temperatura en los tres sitios de muestreo mediante sonda digital YSI 85 y como complemento se utilizó la serie de tiempo de la base de datos del Observatorio Oceanográfico Digital de Venezuela (Klein, E. \& J.C. Castillo, 2009) para establecer las épocas de surgencia (temperatura del agua de $23^{\circ} \mathrm{C}$ a $26^{\circ} \mathrm{C}$ ) y relajación (temperatura del agua de $27^{\circ} \mathrm{C}$ a $30^{\circ} \mathrm{C}$ ).

Una vez obtenidos los datos del número de ovicápsulas, número de especímenes, tallas y sexo se aplicó un análisis de la varianza (ANOVA) para cada una de las variables (abundancia total, abundancia de hembras mayores a $60 \mathrm{~mm}$, fecundidad y estructura de tallas) con la finalidad de encontrar diferencias significativas entre los tres sitios muestreados. En algunos casos se realizó una prueba a posteriori de Tukey para verificar cuál de los sitios estaría generando las diferencias.

\section{RESULTADOS}

Las poblaciones de Voluta musica demostraron tener una densidad de $0,09 \mathrm{ind} / \mathrm{m}^{2}$ $( \pm 7,99)$ en Isla Caribe; $0,04 \mathrm{ind} / \mathrm{m}^{2}( \pm 3,68)$ en Isla Lobos y de $0,10 \mathrm{ind} / \mathrm{m}^{2}( \pm 10,64)$ en Bajo Cuspe. La abundancia varió de 5 a 30 ind $/ 120 \mathrm{~m}^{2}$ dependiendo del sitio y de la fecha en que se 

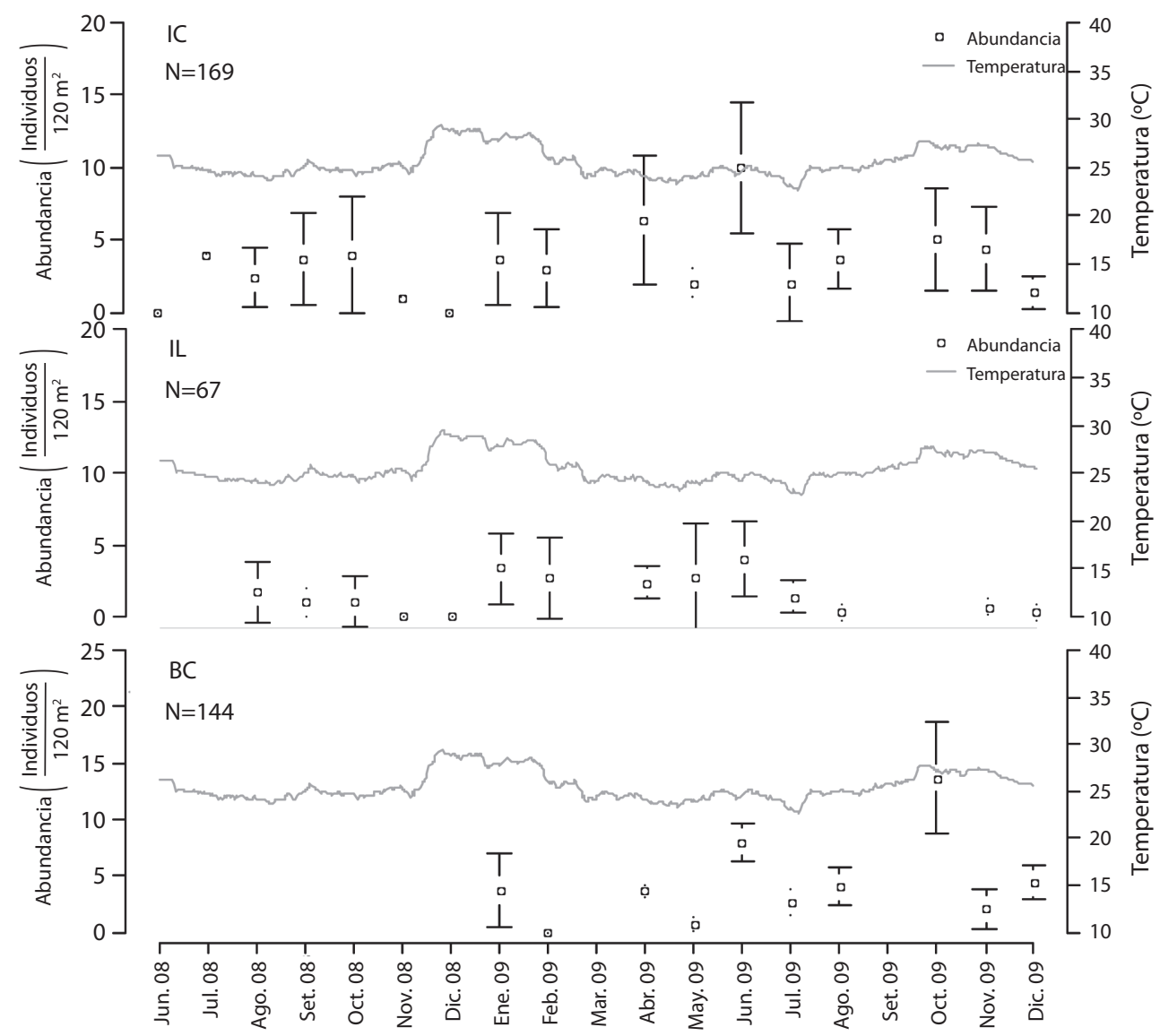

Fig. 1. Comparación de la abundancia de V. musica en los tres sitios de muestreo a lo largo de dos años (2008-2009). IC: Isla Caribe; IL: Isla Lobos; BC: Bajo Cuspe.

Fig 1. Comparison of V. musica abundance in three sites during 2008-2009. IC: Isla Caribe; IL: Isla Lobos; BC: Bajo Cuspe.

tomaron las muestras (Fig. 1). De acuerdo al análisis de la varianza existen diferencias significativas en la abundancia entre los sitios $(\mathrm{F}=7.77 ; \mathrm{p}<0.05)$ y entre los meses del año a excepción de Isla Lobos $(\mathrm{p}<0.05)$.

La prueba a posteriori mostró que la abundancia es igual para Bajo Cuspe e Isla Caribe (Tukey, $\mathrm{p}=0.39$ ) pero distinta y menor en Isla Lobos ( $\mathrm{p}=0.008$; 0.0149).

El análisis de la abundancia de hembras maduras (mayores a $60 \mathrm{~mm}$ de largo) demostró que existen diferencias significativas entre los tres sitios y entre los meses del año $(\mathrm{p}<0,05)$ (Fig. 2). Igualmente, existen diferencias significativas entre los sitios y entre los meses en cuanto al número de ovicápsulas $(\mathrm{p}<0,001)$, siendo Isla Caribe el sitio que genera la diferencia (Tukey, $\mathrm{p}<0,001$ ) con un mayor numero de ovicápsulas en relación a los otros dos sitios (Fig. 3).

La fecundidad de $V$. musica estimada no evidenció diferencias significativas entre sitios ni entre los meses $(p>0,5)$. Los datos indican que la fecundidad de $V$. musica varió de 1 a 32 en Isla Caribe mientras que en Isla Lobos fue de 1 a 12 y en Bajo Cuspe de 1 a 8 ovicápsulas/ hembra/mes (Fig. 3). 


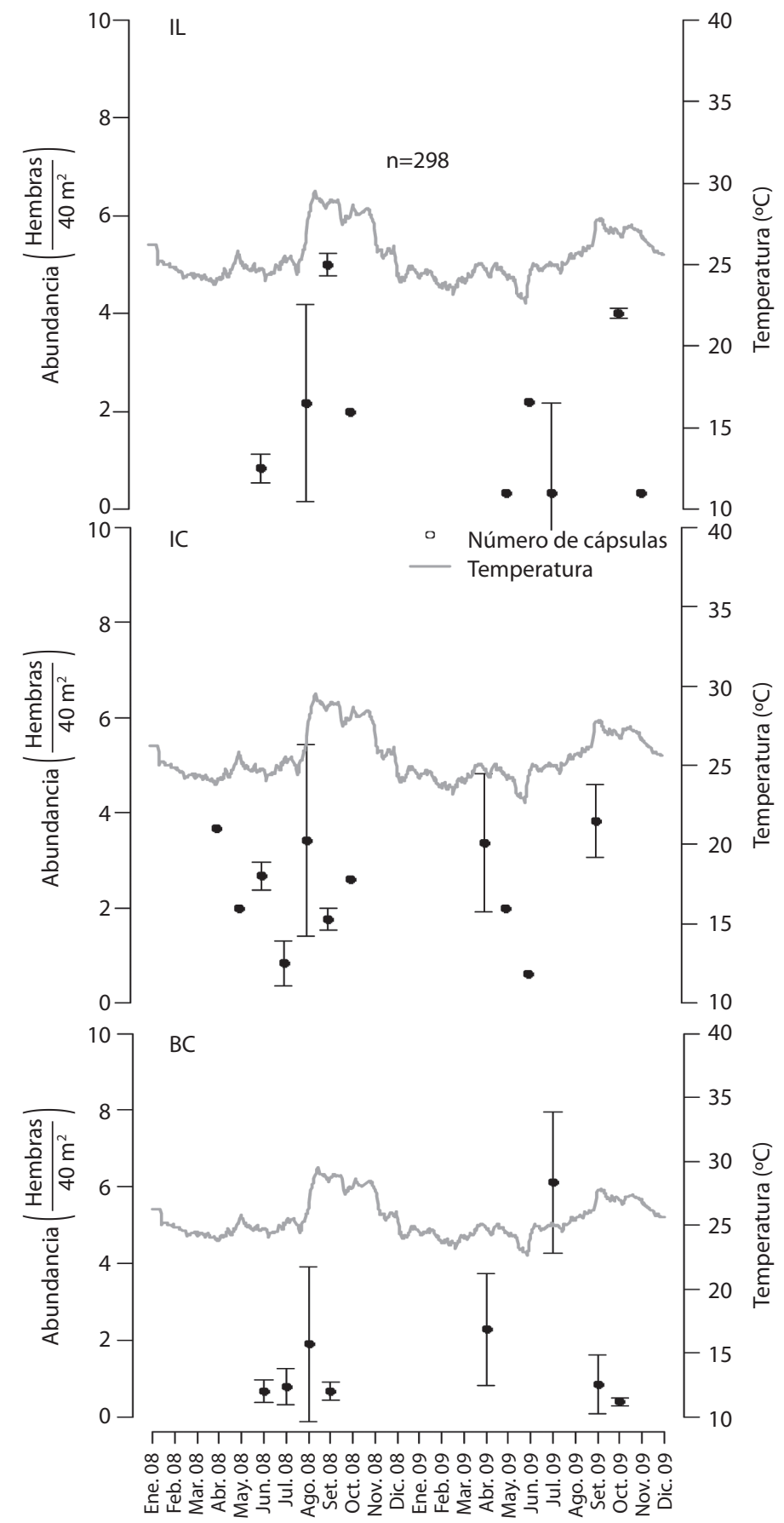

Fig. 2. Abundancia de hembras maduras en tres sitios a lo largo de dos años. IC: Isla Caribe; IL: Isla Lobos; BC: Bajo Cuspe. Fig. 2. Abundance of mature female specimens in three sites during two years. IC: Isla Caribe; IL: Isla Lobos; BC: Bajo Cuspe. 


\section{Isla Caribe}

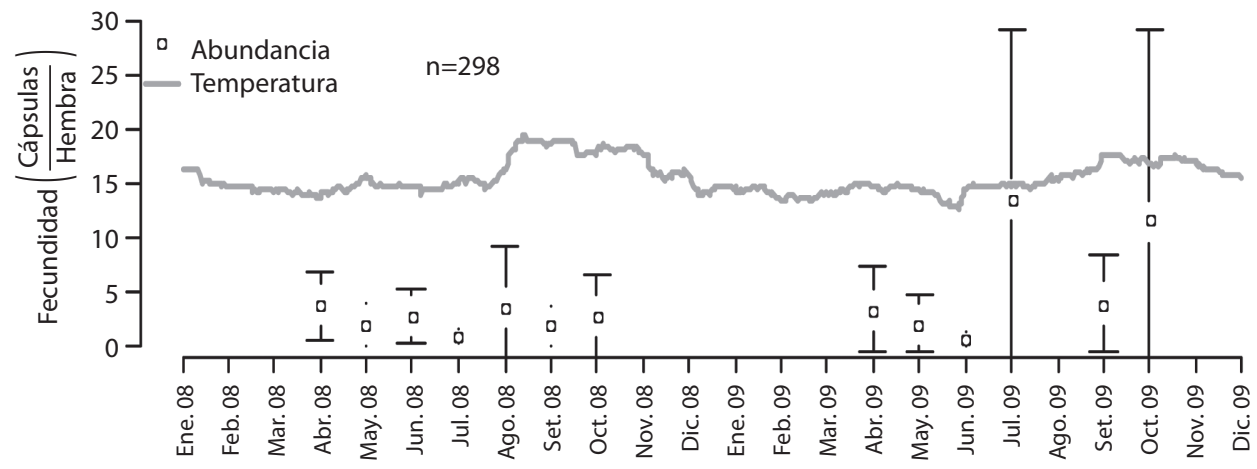

Bajo Cuspe

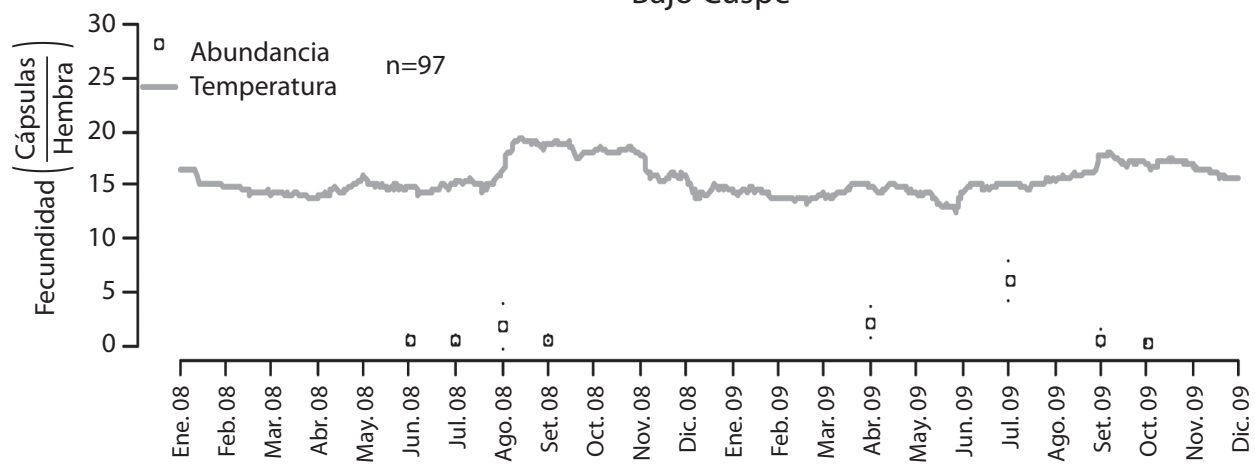

Isla Lobo

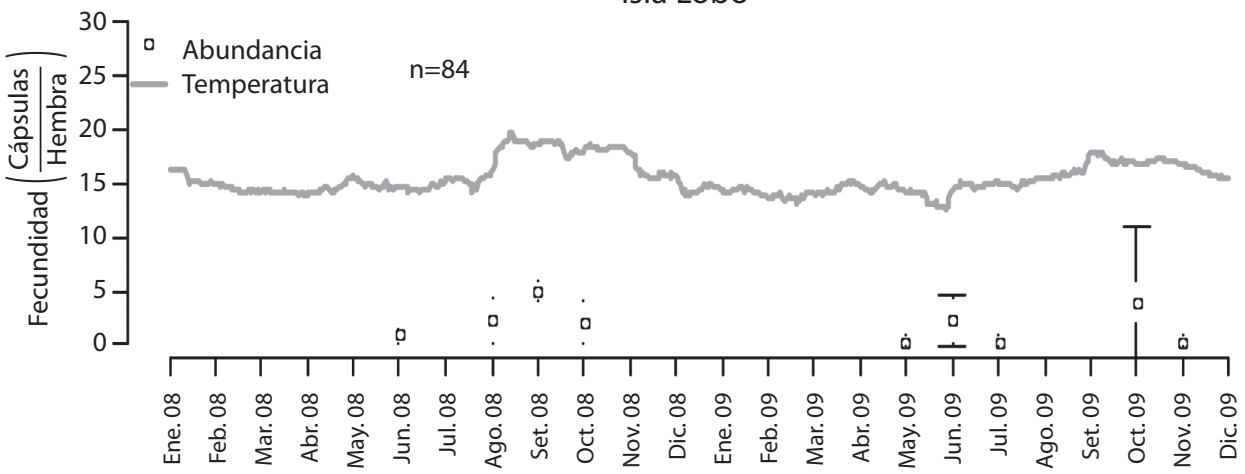

Fig. 3. Número de ovicápsulas por hembra/mes en tres sitios.

Fig. 3. Ovicapsules per female/month in three sites. 
La talla de los individuos medida como largo total de la concha varió de 38,7 a $95 \mathrm{~mm}$ en Isla Caribe, de 34 a $88 \mathrm{~mm}$ en Isla Lobos, y de 34,4 a $89 \mathrm{~mm}$ en Bajo del Cuspe. La talla promedio del largo total de la concha de la población, sin distinción de sexos, fue de: $70,6 \mathrm{~mm}$ en Isla Caribe; $67,25 \mathrm{~mm}$ en Isla Lobos y de $68,4 \mathrm{~mm}$ en Bajo del Cuspe. La comparación de tamaño entre los sexos en cada uno de los sitios indica que los machos son significativamente mas pequeños que las hembras $(\mathrm{p}<0,05)$. No se hallaron individuos menores a $30 \mathrm{~mm}$ de largo total, salvo uno cuya talla fue de $9 \mathrm{~mm}$, encontrado en Isla Caribe (Fig. 4). Se encontraron diferencias significativas en relación al tamaño de los individuos entre los tres sitios $(\mathrm{p}<0.05)$. En una prueba a posteriori se obtuvo que los individuos de Isla Caribe son

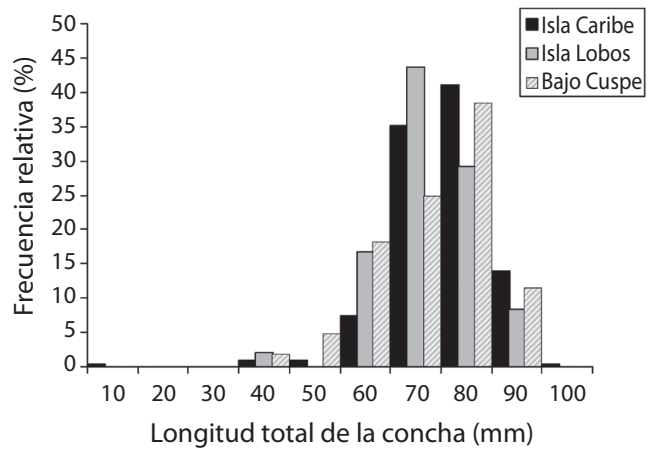

Fig. 4. Estructura de tallas de $V$. musica en tres sitios. Fig. 4. V. musica size structure in three sites.

más grandes en cuanto a la longitud la concha (Tukey, IC >BC > IL, p=0.34; 0.39 y 0.045).

\section{DISCUSION}

Se conoce poco sobre la densidad y abundancia de caracoles de la familia Volutidae, en el caso de Odontocymbiola magellanica, de las costas norpatagónicas, se ha demostrado que a pesar de pasar gran parte del tiempo enterrada tiene cierta capacidad de desplazamiento. No existen grandes bancos en las zonas donde se halla esta especie, sin embargo se encuentran agregaciones de individuos en las épocas reproductivas donde la densidad se calculó en $0,09 \mathrm{ind} / \mathrm{m}^{2} \quad\left(18 \mathrm{ind} / 200 \mathrm{~m}^{2}\right)$ siendo la densidad en épocas no reproductivas de $0,015 \mathrm{ind} / \mathrm{m}^{2}$ (3indiv $/ 200 \mathrm{~m}^{2}$ ) (Bigatti 2005). Por otro lado Carranza et al. (2008) analizaron la distribución y ecología de gasterópodos de la plataforma continental uruguaya y la zona exterior del Río de la Plata, en profundidades desde 4 a $62 \mathrm{~m}$ donde el volútido Adelomelon beckii fue representado por un solo espécimen en todos los casos, sugiriendo bajas densidades poblacionales para la zona de estudio. En el presente trabajo las poblaciones de $V$. musica también demostraron tener bajas densidades de individuos lo que sugiere que las especies de la familia Volutidae tienen distribuciones amplias con pocos individuos, pero que tienen tendencia a agruparse en épocas del año donde ocurren los eventos de cópula y oviposición. Tal y como se demuestra en el presente trabajo, $V$. musica presentó diferencias en cuanto a su densidad en función de los meses del año en dos de los sitios. Cuando analizamos la densidad de las hembras maduras se observa una tendencia a incrementar el número de individuos por área en relación a los meses de oviposición: abril, mayo, junio, agosto, septiembre y octubre (ver Fig. 3).

Hay una clara estacionalidad en la oviposición en los tres sitios muestreados siendo los meses de abril a octubre los meses donde las hembras oviponen, lo que a su vez coincidiría con los meses de aguas más cálidas. El número de ovicápsulas totales fue mayor en Isla Caribe. Sin embargo, el número de ovicápsulas por hembra por mes dentro de cada sitio mostró una alta variabilidad, por lo que el resultado del análisis de la varianza no evidencia diferencias significativas entres los sitios ni entre los meses. Las diferencias en el número de cápsulas entre las áreas muestreadas dentro de cada sitio/mes nos estaría indicando que hay otro factor que determina la ubicación de las ovicápsulas y que éstas no se encuentran presentes en toda el área muestreada. Varios autores han descrito las cápsulas de $V$. musica las cuales son 
colocadas dentro de conchas vacías de bivalvos (Clench \& Turner 1970, Gibson-Smith 1973, von Cosel 1976, Penchaszadeh \& Miloslavich 2001). En algunos casos se encontraron cochas vacías de Atrina seminuda con hasta 30 ovicápsulas adheridas mientras que otras especies de bivalvos como Arca zebra, Codakia sp, y Perna perna, contenían de dos a cuatro ovicápsulas adheridas. Para un próximo estudio, se sugiere considerar el tipo de sustrato disponible para la oviposición en cada uno de los sitios con el fin de minimizar las diferencias que esto podría generar en las determinaciones de abundancia de ovicápsulas dentro de cada mes/sitio.

La estructura de tallas que se obtuvo incluye organismos mayores a $34 \mathrm{~mm}$ lo cual se podría explicar por el hecho de que son organismos crípticos, viven enterrados en el sustrato y solamente exhiben el sifón inhalante apenas unos milímetros fuera del sedimento. Es sabido que la mayoría de los volútidos viven enterrados emergiendo en busca de alimento o en el momento de reproducirse (Clench \& Turner 1964, Clench \& Turner 1970, Poppe \& Goto 1992), esto hace que sea muy difícil encontrar individuos pequeños con la metodología aplicada.

Morton (2006) describe que en ciertas localidades de Hong Kong en donde existe una pesca artesanal intensiva, el murícido Ergalatax contractus es mas abundante que en otros lugares en donde no existe tal actividad, siendo éste un depredador generalista que ocasionalmente consume carroña. Los gasterópodos carroñeros parecen verse favorecidos por los ambientes perturbados (Britton \& Morton 1994, Taylor 1994, Morton 1995, Ramsay et al. 1997, 1998, Morton 2006) debido a una mayor disponibilidad de alimento en forma de carroña. En el presente trabajo se observan individuos de $V$. musica de tallas más grandes en Isla Caribe y con mayor número de oviposturas, probablemente debido a que los individuos de esa población aprovechan la energía que provee el material de desecho de la pesca artesanal compuesto principalmente por pescado, moluscos y crustáceos. Otros trabajos que han reportado a $V$. musica como especie carrroñera son von Cosel (1976) y A.C. Peralta (en prep.), este último determinó que esta especie sea un depredador generalista que presenta hábitos carroñeros, ante la presencia de los descartes de la actividad pesquera. Estos resultados se asemejan a lo observado en los volútidos Adelomelon ancilla (Bigatti et al. 2009) y $O$. magellanica de la Patagonia argentina (Bigatti et al. 2010), donde se observó que consumen principalmente presas vivas y ocasionalmente carroña (Bigatti et al. 2010).

\section{AGRADECIMIENTOS}

Centro de Investigaciones Ecológicas de Guayacán (Universidad de Oriente) por el apoyo en las salidas de campo. A Eduardo Klein por su valiosa orientación en el análisis de los datos y Emanuel Valero por su colaboración en el uso de paquetes estadísticos. Al Decanato de Investigación y Desarrollo-USB, y al Decanato de Postgrado-USB por el financiamiento otorgado.

\section{RESUMEN}

Teniendo en cuenta la intensa actividad pesquera artesanal y subsecuente fuente adicional de alimento como carroña en Isla Caribe, se esperaría un efecto sobre algunos parámetros poblacionales de V. musica como: mayor número de ovicápsulas, individuos de tallas mayores y densidades de caracoles adultos mayores. Con el presente trabajo se desea conocer la abundancia, estructura de talla y fecundidad de Voluta musica en tres sitios de la costa norte de la Península de Araya en las que existen actividades de pesca diferenciales. Se realizaron muestreos mensuales entre 2008 y 2009 en Isla Caribe, Isla Lobos y Bajo Cuspe, en cada uno con 3 áreas de $40 \mathrm{~m}^{2}$. La abundancia varió de 5ind $/ 120 \mathrm{~m}^{2}$ a $30 \mathrm{ind} / 120 \mathrm{~m}^{2}$, con diferencias significativas entre los sitios $(\mathrm{F}=.77 ; \mathrm{p}<0,01)$, siendo igual para Bajo Cuspe e Isla Caribe $(\mathrm{p}=0,39)$ pero distinta y menor en Isla Lobos, $(p=0,008 ; 0,0149)$. Los individuos de Isla Caribe demostraron ser más grandes $(\mathrm{p}=0,045)$. Existen diferencias significativas entre sitios y entre meses en el número de ovicápsulas $(\mathrm{p}<0,01)$, siendo Isla Caribe el sitio con mayor abundancia de ovicápsulas $(\mathrm{p}<0,01)$. Esto sugiere que el alimento suplementario en forma de carroña podría incrementar el potencial reproductivo de la población en Isla Caribe.

Palabras claves: Volutidae, pesca artesanal, ovicápsulas, fecundidad, Caribe Sur. 


\section{REFERENCIAS}

Bigatti, G. 2005. Anatomía, Ecología y Reproducción del caracol rojo Odontocymbiola magellanica (Gastropoda: Volutidae) en golfos norpatagónicos. Tesis de $\mathrm{Ph}$. D., Universidad de Buenos Aires, Facultad de Ciencias Exactas y Naturales, Buenos Aires, Argentina.

Bigatti, G., Carlos .J.M. Sánchez Antelo, P. Miloslavich \& Pablo E. Penchaszadeh. 2009. Adelomelon ancilla: a predator neogastropod in Patagonia benthic communities. Nautilus 123: 159-165.

Bigatti, G., Hernán Sacristán, María C. Rodríguez, Carlos A. Storz \& Pablo E. Penchaszadeh. 2010. Diet, prey narcotization and biochemical composition of salivary glands secretions of the volutid snail Odontocymbiola magellanica. J. Mar. Biol. Assoc. UK 90: 1-9.

Britton, J.C. \& B. Morton. 1994. Marine carrion and scavengers. Oceanogr. Mar. Biol. Ann. Rev. 32: 369-434.

Carranza, A., F. Scarabino \& L. Ortega (2008). "Distribution of Large Benthic Gastropods in the Uruguayan Continental Shelf and Río de la Plata Estuary.” Journal of Coastal_Research 24: 161-168.

Clench, W. \& R.D. Turner. 1964. The subfamilies Volutinae, Zidoninae, Odontocymbiolinae and Calliotectinae in the Western Atlantic. Johnsonia 4: 129-179.

Clench, W. \& R.D. Turner. 1970. The family volutidae in the Western Atlantic. Johnsonia 4: 369-392.

Gibson-Smith, J. 1973. The genus Voluta (Mollusca, Gastropoda) in Venezuela with description of two new species. Geos 20: 65-73.

Morton, B. 1995. Perturbated soft intertidal and subtidal marine communities in Hong Kong: the significance of scavenging gastropods, p. 1-15. In X.G.Z.B. Morton, Z. Renlin, P. Jinpei \& C. Guoxiong (eds). The Marine Biology of the South China Sea II. Proc. 2nd Int. Conf. Mar. Biol. South China Sea, China.
Morton, B. 2006. Scavenging behaviour by Ergalatax contractus (Gastropoda: Muricidae) and intercations with Nassarius nodifer (Gastropoda: Nassariidae) in the Cape d'Aguilar Marine Reserve, Hong Kong. J. Mar. Biol. Assoc. UK 86: 141-152.

Penchaszadeh, P. \& P. Miloslavich. 2001. Embryonic stages and feeding substances of the South American volutid Voluta musica (Caenogastropoda) during intracapsular development. Amer. Malacol. Bull. 16: 21-23.

Poppe, G. T. and Y. Gotto (1992). Volutes. Ancona, Italia.

Ramsay, K., M, Kaiser, P.G. Moore \& R.N. Hughes. 1997. Consumption of fisheries discardas by benthic scavengers: utilization of energy subsidies in different marine habitats. J. Anim. Ecol. 66: 884-896.

Ramsay, K., M.J. Kaiser \& R.N. Hughes. 1998. Responses of benthic scavengers to fishing disturbance by towed gears in different habitats. J. Exp. Mar. Biol. Ecol. 224: 73-89.

Rodríguez, J.P. \& F. Rojas-Suárez. 2008. Libro Rojo de la Fauna Venezolana. Provita, Caracas, Venezuela.

Taylor, J.D. 1994. Sublittoral benthic gastropods around southern Hong Kong, p. 479. In B. Morton (ed.). The Malacofauna of Hong Kong and Southern China III. Hong Kong Univ. Press, Hong Kong.

von Cosel, R. 1976. Contribución al conocimiento del género Voluta Linné, 1758 (Prosobranchia) en la costa del Caribe de Colombia. Mitt. Inst. ColomboAlemán Invest. Cient. 8: 83-104.

\section{Referencias de internet}

Klein E. y J.C. Castillo, 2009. Observatorio Oceanográfico Digital del Mar Venezolano. Laboratorio de Sensores Remotos, Centro de Biodiversidad Marina INTECMAR-USB. http://ood.cbm.usb.veObservatorio Oceanográfico Digital de Venezuela (http://ood. cbm.usb.ve/historial/sst_noaa/) 